\title{
A SPECTRAL CHARACTERIZATION OF ABSOLUTELY NORMING OPERATORS ON S. N. IDEALS
}

\author{
SATISH K. PANDEY
}

Abstract. The class of absolutely norming operators on complex Hilbert spaces of arbitrary dimensions was introduced in [6] and a spectral characterization theorem for these operators was established in [11]. In this paper we extend the concept of absolutely norming operators to various symmetric norms. We establish a few spectral characterization theorems for operators on complex Hilbert spaces that are absolutely norming with respect to various symmetric norms. It is also shown that for many symmetric norms the absolutely norming operators have the same spectral characterization as proven earlier for the class of operators that are absolutely norming with respect to the usual operator norm. Finally, we prove the existence of a symmetric norm on the algebra $\mathscr{B}(\mathscr{H})$ with respect to which even the identity operator does not attain its norm.

Mathematics subject classification (2010): Primary 47B07, 47B10, 47L20, 47A10, 47A75, Secondary 47A05, 47L07, 47L25, 47B65, 46L05.

Keywords and phrases: Symmetric norms, s. n. functions, s. n. ideals.

\section{REFERENCES}

[1] M. D. Acosta, Denseness of norm-attaining operators into strictly convex spaces, Proceedings of the Royal Society of Edinburgh: Section A Mathematics 129 (1999), no. 06, 1107-1114.

[2] M. D. Acosta, F. J. Aguirre, And R. PAy, A new sufficient condition for the denseness of norm attaining operators, Journal of Mathematics 26 (1996), no. 2.

[3] M. D. Acosta And M. R. Galan, Norm attaining operators and reflexivity, Rend. Circ. Mat. Palermo 56 (1998), no. 2 Suppl, 171-177.

[4] M. D. Acosta And C. RUIZ, Norm attaining operators on some classical Banach spaces, Mathematische Nachrichten 235 (2002), 17-28.

[5] F. J. AgUIRRE, Norm-attaining operators into strictly convex Banach spaces, Journal of mathematical analysis and applications 222 (1998), no. 2, 431-437.

[6] X. Carvajal And W. Neves, Operators that achieve the norm, Integral Equations and Operator Theory 72 (2012), no. 2, 179-195.

[7] J. B. Conway, A course in operator theory, American Mathematical Society, Providence, R. I, 2000.

[8] K. FAN, Maximum properties and inequalities for the eigenvalues of completely continuous operators, Proceedings of the National Academy of Sciences 37 (1951), no. 11, 760-766.

[9] I. C. GOHBERG AND M. G. KREIN, Introduction to the theory of linear nonselfadjoint operators, American Mathematical Society, Providence, R. I, 1969.

[10] G. S. MudholkAR And M. Freimer, A structure theorem for the polars of unitarily invariant norms, Proceedings of the American Mathematical Society 95 (1985), no. 3, 331-337.

[11] S. K. Pandey And V. I. Paulsen, A spectral characterization of $\mathscr{A} \mathscr{N}$ operators, Journal of the Australian Mathematical Society (2016), 1-23.

[12] S. K. PANDEY, A spectral characterization of absolutely norming operators on s. n. ideals, arXiv preprint arXiv:1610.02095 (2016).

[13] J. R. Partington, Norm attaining operators, Israel Journal of Mathematics 43 (1982), no. 3, 273276.

[14] G. Ramesh, Structure theorem for $\mathscr{A} \mathscr{N}$-operators, Journal of the Australian Mathematical Society 96 (2014), no. 3, 386-395. 
[15] W. SCHACHERMAYER, Norm attaining operators and renormings of Banach spaces, Israel Journal of Mathematics 44 (1983), no. 3, 201-212.

[16] W. SCHACHERMAYER, Norm attaining operators on some classical Banach spaces, Pacific Journal of Mathematics 105 (1983), no. 2, 427-438.

[17] S. SHKARIN, Norm attaining operators and pseudospectrum, Integral Equations and Operator Theory 64 (2009), no. 1, 115-136. 